\title{
Anomalous subaortic position of the brachiocephalic vein (innominate vein): an echocardiographic study
}

\author{
Jung Yun Choi, Mi Jin Jung, Young Hwi Kim, Chung Il Noh, Yong Soo Yun
}

\begin{abstract}
In $24(0.98 \%)$ of 2457 patients with congenital heart disease the brachiocephalic vein was in an anomalous position below the aortic arch. This is a much higher proportion of such cases than reported so far. This high frequency may arise from differences in the study population and the method of diagnosis. This venous anomaly was more common in patients with tetralogy of Fallot or ventricular septal defect with pulmonary atresia. Patients with the venous anomaly were more likely to have a right aortic arch.

The anomalous course of the brachiocephalic vein from the neck to the junction of the superior vena cava was shown by cross sectional echocardiography. In doubtful cases, Doppler study usually clarified the anatomical arrangement.
\end{abstract}

The left innominate vein extends from the junction of the left internal jugular vein and the left subclavian vein to the superior vena cava. It runs above and in front of the aortic arch and in front of the left subclavian, left common carotid, and innominate arteries. In atrial situs inversus the arrangement of the systemic veins is usually the mirror image of this.

Rarely the left brachiocephalic vein takes an anomalous course below the aortic arch. This was first described by Kershner a hundred years ago. ${ }^{1}$ Smallhorn et al described echocardiographic findings of this anomaly ${ }^{2}$ and Gerlis and Ho recently reviewed a total of 25 patients including three of their own. ${ }^{3}$ Though this anomaly is not clinically important, it may be confused with the pulmonary artery in cross sectional echocardiography especially in the patients with very hypoplastic or atretic central pulmonary arteries.

Gerlis and Ho thought that this venous anomaly was very rare-about one in 500 congenitally malformed hearts. But we found that this lesion is not rare in certain cardiac defects. We found 24 cases among 2457 echocardiograms of congenital heart disease in 21 months and we report the incidence and associated congenital heart diseases in this malformation.

\section{Patients and methods}

We reviewed echocardiograms obtained between January 1988 and September 1989 in the department of paediatrics, Seoul National University Children's Hospital to find out the total number of new patients with congenital heart disease, the types of cardiac defect, and the patients who had an anomalous brachiocephalic vein running below the aortic arch. If necessary, the diagnosis of the cardiac defects was updated after cardiac catheterisation or cardiac surgery.

The diagnosis of a subaortic brachiocephalic vein was mainly based on the cross sectional echocardiographic and Doppler findings. In the first few cases cardiac catheterisation confirmed the anomalous position of the brachiocephalic vein. Some cases had surgical confirmation too.

We used the $\chi^{2}$ test or Fisher's exact test for statistical analysis and a $p$ value $<0.05$ was regarded as significant.

\section{Results}

Twenty four $(0.98 \%)$ patients with an anomalous subaortic brachiocephalic vein were found among 2457 patients with congenital heart disease. Twenty three patients had a normally arranged atria and the left brachiocephalic veins were below the aortic arch. One patient had atrial inversion, a left sided superior vena cava, and a right brachiocephalic vein associated with a complex intracardiac defect. In this patient the right brachiocephalic vein was below the left aortic arch and intracardiac defects consisted of discordant atrioventricular connection with single atrioventricular valve, single aorta from right ventricle, and pulmonary atresia. Table 1 shows the frequency with which the venous anomaly was found in several cardiac malformations. It was very common in cases of tetralogy of Fallot and ventricular septal defect with pulmonary atresia. Table 2 summarises the clinical characteristics and the frequency of right aortic arch in the patients who had either tetralogy of Fallot or ventricular septal defect with

Table 1 Frequency of anomalous brachiocephalic vein in some cardiac malformations

\begin{tabular}{llll}
\hline $\begin{array}{l}\text { Cardiac } \\
\text { lesion }\end{array}$ & $\begin{array}{l}\text { No with } \\
\text { cardiac defect }\end{array}$ & $\begin{array}{l}\text { No with } \\
\text { anomalous vein }\end{array}$ & $\begin{array}{l}\text { Frequency } \\
(\%)\end{array}$ \\
\hline VSD & 1042 & 1 & $0 \cdot 01$ \\
TOF & 177 & 10 & $5 \cdot 7$ \\
VSD + PA & 57 & 10 & 17.5 \\
Others & 1181 & $3^{\star}$ & \\
Total & 2457 & 24 & 0.98
\end{tabular}

*Each patient had truncus arteriosus, complete transposition of the great arteries, and a complex anomaly with inverted

atrial situs.
PA, pulmonary atresia; TOF, tetralogy of Fallot; VSD, ventricular septal defect. 
Table 2 Clinical characteristics and laterality of aortic arch in patients with subaortic brachiocephalic vein

\begin{tabular}{|c|c|c|c|c|}
\hline $\begin{array}{l}\text { Cardiac } \\
\text { defect } \\
(n)\end{array}$ & $\begin{array}{l}\text { Age } \\
\text { (mean) } \\
\text { (range) }\end{array}$ & $\begin{array}{l}\text { Sex } \\
\text { ratio } \\
(M: F)\end{array}$ & $\begin{array}{l}\text { Proportion } \\
\text { of right } \\
A A(\%)\end{array}$ & $\begin{array}{l}\text { Proportion of } \\
\text { right } A A \text { in } \\
\text { those without } \\
\text { venous anomaly (\%) }\end{array}$ \\
\hline TOF & 1 yr 9 mnth & 2.3:1 & 80 & $20 \cdot 4$ \\
\hline$\underset{(10)}{\operatorname{VSD}}+\mathrm{PA}$ & $\begin{array}{l}1 \text { yr } 9 \text { mnth } \\
\text { ( } 3 \text { mnth-10 yr) }\end{array}$ & $2 \cdot 3: 1$ & 50 & $21 \cdot 3$ \\
\hline
\end{tabular}

AA, aortic arch. See footnote to table 1 for other abbreviations.

Figure 1 Frontal view from suprasternal notch showing the subaortic left brachiocephalic vein (arrows) running in front of the left subclavian artery and below the left innominate artery and aortic arch. Ao, aorta; $I A$, innominate artery.

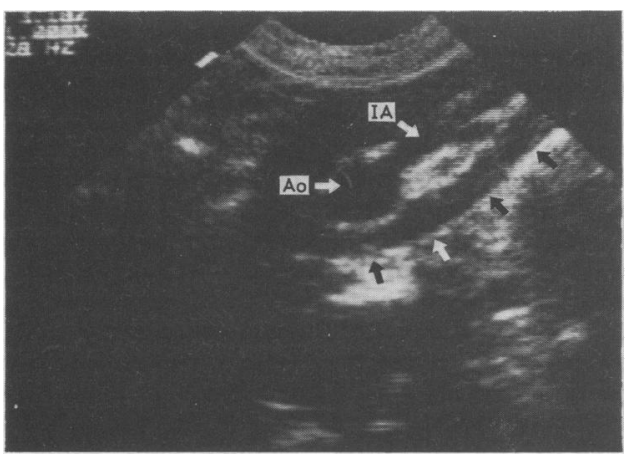

Figure 2 Suprasternal para-sagittal view shows two circular structures below the aortic arch. The upper circle (arrows) is the left brachiocephalic vein and the lower one is the right pulmonary artery ( $R P A$ ).

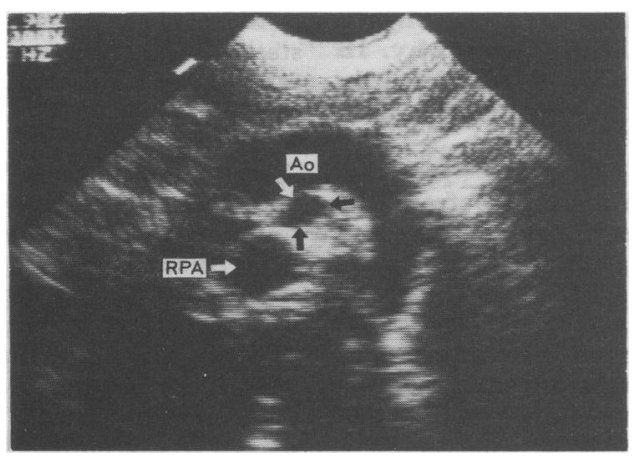

Figure 3 Doppler spectrum $(B)$ recorded from the left brachiocephalic vein $(A)$ and showing low velocity venous flow toward the heart. associated pulmonary atresia and the brachiocephalic vein in an anomalous position. The table also shows how often a right aortic arch was diagnosed in patients with the same cardiac defects but without an anomalous brachiocephalic vein during the same period. A right aortic arch was significantly more common in patients with an anomalous vein than in those without ( $p<0.05$ in tetralogy of Fallot and $p<0.05$ in ventricular septal defect with pulmonary atresia).

Five patients had a persistent ductus arteriosus. Three of them had a ventricular septal defect, pulmonary atresia, a right aortic arch, and a left ductus arteriosus arising from the left innominate artery. Another patient had tetralogy of Fallot, left aortic arch, and a left ductus arteriosus and the remaining patient had atrial situs inversus, a left aortic arch, right ductus arteriosus from the right innominate artery, and the intracardiac defects described earlier. The ductus arteriosus always passed in front of the anomalous brachiocephalic vein. Ten of the 19 patients without a persistent ductus arteriosus had an operation. But in the surgical descriptions of these operations there was no comment of the presence of a ligamentum arteriosum or its relation to the anomalous vein.

Cross sectional echocardiography in the suprasternal frontal plane showed the anomalous left brachiocephalic vein between the right pulmonary artery (below) and the aorta (above). This anomalous vein could usually be traced to the left side of the neck when the transducer is tilted leftwards and anteriorly. In patients with a right aortic arch and left innominate artery, the anomalous vein descended from the left side of the neck and crossed the left subclavian artery anteriorly, ran under the left innominate artery, and then joined the superior vena cava (fig 1 ). When the ductus arteriosus arose from the left innominate artery, slight tilting of the transducer showed the relation between the vein and the ductus. But in patients with a left aortic arch this vein ran in front of the left common carotid and left subclavian arteries so that it was very difficult to get a simultaneous demonstration of this vein and head and neck arteries.

The parasagittal view showed two circles below the aortic arch. The upper one is the anomalous vein and the lower one is the right pulmonary artery (fig 2). Doppler examination showed the typical venous flow signal toward the heart (fig 3).

\section{Discussion}

The anomalous subaortic course of the left brachiocephalic vein was first described about a hundred years ago. ${ }^{1}$ Gerlis and Ho reviewed 25 cases, including three of their own patients. ${ }^{3}$ They reported that all these patients had an anomalous left brachiocephalic vein. In our study we found one patient who had a right brachiocephalic vein below the left aortic arch with an inverted atrial arrangement. Gerlis and Ho estimated the incidence of this malformation to be about $0 \cdot 2 \%$ in their necropsy series. 
In our clinical study we found the anomaly in $0.98 \%$ of patients. This difference may be due to the difference in the study population and the method of diagnosis. The two echocardiographers in our laboratory are well aware of this anomalous position of the brachiocephalic vein and the echocardiographic findings when this lesion is present are so characteristic that they are unlikely to miss or overdiagnose this lesion. The lower incidence found in the necropsy series may have been lower because tetralogy of Fallot and ventricular septal defect with pulmonary atresia, both conditions in which the venous anomaly is common, do not have a high mortality rate. Therefore these two cardiac malformations were less common in the necropsy series. Furthermore this lesion might be missed during the routine examinations.

Because we do not know of another clinical study of the incidence of this venous anomaly it is impossible to tell whether there is a racial variation.

Smallhorn et al described the echocardiographic findings associated with anomalous brachiocephalic vein and cautioned that this lesion might be confused with the central pulmonary artery. ${ }^{2}$ The echocardiographic findings in our cases are almost the same as those described by Smallhorn et $a l$, and in addition, we found that this vein was usually traceable to the left side of the neck. If this vein is properly traced, it is not likely to be confused with other vessels, and in doubtful cases Doppler study usually clarifies the situation.

The most frequently associated cardiac defects were tetralogy of Fallot and ventricular septal defect with pulmonary atresia, both of which were present in 20 of the 24 cases with the venous anomaly. Another two patients had conotruncal anomalies-truncus arteriosus or transposition of the great arteries. There were 22 conotruncal anomalies in total. This association of anomalous subaortic brachiocephalic vein with the conotruncal anomalies seems to be highly significant. In addition, a right aortic arch was more common in patients with the venous anomaly than in those without. The embryological origins of the anomalous vein have been reviewed ${ }^{3}$ but the relation between the abnormal position of the brachiocephalic vein, conotruncal cardiac anomalies, and the right aortic arch has yet to be established.

This study was partly supported by the Seoul National University Children's Hospital Research Fund.

1 Kershner L. Zur Morphologie der Vena Cava Inferior. Anat
Anz 1888;3:808-23. (Cited in reference 3 by Gerlis and Anz

2 Smallhorn JF, Zielinsky P, Freedom RM, Rowe RD. Abnormal position of the brachiocephalic vein. Am $j$

3 Gerlis LM, Ho SY. Anomalous subaortic position of the brachiocephalic (innominate) vein: a review of published reports and report of three new cases. Br Heart $J$ 1989;61:540-5. 